\title{
REMOTE MAINTENANCE OF COMPACT IGNITION TOKAMAK EX-VESSEL SYSTEMS*
}

R. E. DePew and D. Macdonald

Central Engineering

Oak Ridge National Laboratory

Oak Ridge, Tennessee 37831-6353

To be presented at the

13th Symposium on Fusion Engineering

Knoxville, Tennessee

October 2-6, 1989

*Research sponsored by the Office of Fusion Energy. U. S. Department of Energy, under contract DE-AC05-84OR21400 with Martin Marietta Energy Systems, Inc. 


\author{
R. E. DePew and D. Macdonald \\ Central Engineering \\ Oak Ridge National Laboratory, Oak Ridge, Tennessee 37831-6353
}

\begin{abstract}
$\underline{\text { Absiract }}$
The use of deuterium-tritium (D-T) fuel in the Compact Ignition Tokamak (CIT) will require applying remote handling technology for ex-vessel maintenance and replacement of machine components. Highly activated and contaminated components of the fusion device's auxiliary systems, such as diagnostics and RF heating, must be replaced using remotely operated maintenance equipment in the test cell.
\end{abstract}

Throughout the CIT remote maintenance (RM) studies conducted to date, computer modeling has been used extensively to investigate manipulator access in these complex, tightly packs, and cluttered surroundings. A recent refinement of computer modeling involves the use of an intelligent engineering work station for realtime interactive display of task simulations. This paper discusses the use of threedimensional (3-D) kinematic computer models of the CIT machines that are proving to be powerful tools in our efforts to evaluate $R M$ requirements. This presentation includes a video-taped simulation of remote replacement of a plasma viewing assembly. The simulation illustrates

\footnotetext{
*Research sponsored by the Office of Fusion Energy, U.S. Department of Energy, under contract DE-AC05-84OR21400 with Martin Marietta Energy Systems, Inc.
}

some of the constraints associated with typical RM activities and the ways in which computer modeling enhances the design process.

\section{Introduction}

The CIT fusion device is located in the center cell of the test facility. The machine will operate initially in a nonactivating hydrogen phase for approximately one year. This will permit hands-on repair of equipment that fails during shakedown runs and demonstration of RM operations. Limited access to the test cell may still be possible after deuterium-deuterium (D-D) operations commence; however, once D-T fuel is introduced, personnel access into the center cell of the test facility will be prohibited, and repair and replacement of machine components will be accomplished by remotely operated equipment. Virtually all machine components that interface with the vertical and horizontal ports of the vacuum vessel will be designed for remote replacement and handling. These components will be repaired or packaged for disposal in the hot repair cell. Figure 1 shows the arrangement of the various cells in the shielded facility. The development of the exvessel RM (XVRM) concept for CIT has been the prime responsibility of Oak Ridge National Laboratory (ORNL) for Princeton Plasma Physics Laboratory and the Department of Energy [1].

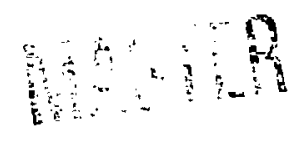


The key element of the test cell maintenance system is a pair of bilateral, force-r iliecting, servomanipulator arms mounted on a telescoping boom supported from an overhead transporter to provide dexterous manipulation throughout the test cell. The system is the primary means for remote operations on the upper vertical ports and the horizontal ports of the machine and on the floor area of the center cell. Replacing equipment in the center cell requires opening the sliding shield roof, decoupling components, and enclosing them in a container to prevent the spread of contamination before removing them to the hot repair cell for repair or disposal. These operations are performed remotely using the servomanipulator and crane hook reaching down from their high bay bridges.

Remote maintenance technology itself is well established, but its application to fusion is underdeveloped. The CIT poses many unique $\mathrm{RM}$ issues, such as 1) high equipment density with limited flexibility in machine configuration; 2) neutron activation of equipment components and facility structures; and 3) delicate tasks to be performed on complex equipment by teleoperation in areas of restricted access and even in blind holes. Remote maintenance requirements must share equal status with fusion requirements on essential machine components that also have a failure potential. A major effort has been made to narrow the gap in technical understanding by thorough interaction between remote maintenance and fusion designers. Regular work sessions are held with all the component designers to integrate RM and equipment design. Prototype hardware mock-ups are being used to demonstrate critical features, and 3-D computer modeling has been used extensively to investigate manipulator access with respect to maximum envelope, reach, and kinematics. The use of real-time graphic simulation of RM tasks, as described below, is proving to be a powerful tool in our efforts to analyze remote repair tasks and verify feasibility.

\section{Graphic Modeling of CIT}

IGRIP (Interactive Graphics Robot Instruction Program) is a computer graphics program from Deneb Robotics, Inc., for workcell layout and simulation. It can be used to model workspaces and kinematic geometry used within a remote facility (manipulators, cranes, transporters, etc.). Because collision avoidance is always an important consideration in RM, IGRIP's collision detection and "near-miss" detection features are proving to be excellent tools. Simulation of an activity or process is a powerful feature of this software. Programs can be written for IGRIP devices to perform tasks within the workspace. This feature allows RM designers to perform detailed maintenance studies in true 3-D geometry.

Devices in IGRIP can be assigned attributes, such as joint limits, joint types (rotational or translational), maximum link speeds, maximum link accelerations, and degrees of freedom (DOF) descriptions. These physical attributes provide the means to realisticaliy model manipulators, cranes, and other RM equipment.

IGRIP allows the CAD operator to view a model in one of several display modes, including flat shading, fancy shading, garaud shading, wire frame, and hidden line. The operator can instantly change from one display mode to another while viewing the model. IGRIP has a single point light source that can easily be moved in space to provide appropriate shading. IGRIP allows the operator to "fly" through the workcell to view the model from any point and orientation. This viewing flexibility provides RM designers/CAD operators with the "right" vantage point for analyzing a design or layout.

The XVRM group is using a Silicon Graphics model 4D80GT stand-alone workstation because of its ability to run the IGRIP simulations at real-time speeds and because the worksta! in can be upgraded to 
provide greater computational power and speed as the CIT RM model gets larger. Several peripherals are attached to the workstation. A Seiko colcr plotter produces shaded color prints on both paper and transparency film. A video peripheral allows IGRIP simulations to be recorded on video tapes for replay during presentations. Visual media from the plotter and video peripheral are used to help communicate concepts to others. The workstation is connected to a host computer system at ORNL, allowing communication and file transfers between the workstation and various other computers. Designs compieted on other CAD systems can be transferred to IGRIP and included in the simulation and analysis. XVRM designers use this communication capability to share data and graphics information with others.

A large portion of the CIT facility has been modeled in IGRIP to date. The entire facility will eventually be included in the model as the detail design is finalized. Shown in Fig. 1, the current facility model includes the center test cell, north and south cells, high bay, hot and contact repair cells, operating gallery, and loading area. The model contains a moderate level of detail such as passageways, doorways, and roof hatches. Details will be added continuously as the project matures.

Figure 2 shows the fusion device, several diagnostics attached to the vertical and horizontal ports, the telescoping-boommounted manipulator system, and the bridge crane system. Kinematics have been assigned to the servomanipulator arms, the manipulator overhead transporter and boom system, the bridge crane system, the sliding shield roof, and the plasma/IRTV diagnostic.

The simulation capabilities of IGRIP hrve been used with the CIT model to depict XVRM activities. These simulations have proved useful in several ways. In conjunction with IGRIP's interference detection, the simulations have allowed XVRM designers to evaluate access constraints and address access problems that might otherwise have gone unnoticed for some time and could not have been evaluated until hardware mock-ups were constructed. The simulations have aided in developing maintenance sequences for exvessel equipment. The simulations also provide a means by which to clearly and succinctly communicate RM ideas and methods to others. This is particularly important when considering the gap in technical understanding between those responsible for designing fusion equipment and those responsible for the $\mathrm{RM}$ of this equipment.

\section{Plasma/IRTV System}

The plasma/IRTV diagnostic will be used for imaging the plasma and diverter region during a pulse and for collecting light from Thomson scattering of laser light by plasma electrons. It may be used for internal inspection of the vacuum vessel if enough light can be provided.

The system, shown in Fig. 3, is rigidly supported, independent of the vacuum vessel, from the test cell floor. A fused silica tube is cantilevered through the horizontal port and supports the forward two mirrors of the system at the mouth of the port. The tube is supported on a pair of ceramic ball bearings, enabling it to rotate around its long axis, thus scanning the field of view about the vacuum vessel interior. The rotary motion is transmitted by a precision rotary vacuum feedthrough driving a ring gear attached to the aft end of the tube. The optical path passes from the forward mirrors through the interior of the tube to a folding mirror mounted in a tee-shaped chamber. Here the path is reflected down to the vacuum window, which is made of fused silica with a 6-in. clear aperture. The vacuum seal at the horizontal port flange is made via a 12-in.-long, 12-in.diam, highly flexible, reentrant bellows to allow movement of the vessel relative to the 
system without damage or misalignment of the system. This bellows also isolates the diagnostic from machine vibration.

Several design features have been provided to simplify RM of the plasma/RTV. A bracket fastened to the top of the plasma/IRTV is bolted to the port flange before the diagnostic is removed from the port to support ind constrain the highly flexible flange beivuws during transit to the hot repair cell. All of the flange bolts are captured and spring-loaded. A telescoping section has been added to the support post to increase accessibility to the flange and IRTV components. The telescoping section is lowered during maintenance of the plasma/IRTV to allow access to the lower flange bolts and better visual access to the entire lower portion of the diagnostic. Because the vacuum vessel will be connected to an offgas system during maintenance periods, it will be under a slight vacuum during removal of the plasma/IRTV. Pneumatic actuators at the top and bottom of the port flange overcome the slight vacuum and separate the plasma/IRTV flange from the port flange to allow removal of the diagnostic from the port. A vacuum isolation valve is located between the tee section and the tube section of the system, so that the window can be changed remotely without breaking vacuum in the vessel. The valve will be closed while the window is being replaced to retain the vacuum inside the vessel and reopened after the new window is installed. Lifting brackets have been added to allow a lifting fixture to be attached to the plasma/IRTV. The motors used to adjust mirrors are designed to be remotely replaced.

A simulation showing the removal of a plasma/IRTV diagnostic was first completed in April 1989. The simulation has been updated periodically to reflect changes in facility and fusion device design and RM requirements. The simulation illustrates the steps required to remotely remove the plasma/IRTV with the manipulator and crane.
The sliding shield roof opens first to allow access to the fusion device components. The manipulator is ? the plasma/KTV and loosens the flange bolts. The crane uses a lifting fixture to support the diagnostic while the floor-mounted telescoping support post is lowered. The crane then transports the plasma/IRTV to the hot repair cell. The shield roof is then closed, and the crane and manipulator are returned to the high bay. The modeling portion of the initial simulation required about $\mathbf{1 2 0}$ work-hours to complete, and the programming portion required another 80 work-hours of concentrated effort.

\section{Conclusions}

The XVRM group plans far-reaching use of graphics modeling in future CIT work. Detailed test layouts in IGRIP should disclose interferences among fusion device components and diagnostics, allowing such problems to be solved during the initial design phase. Remote maintenance concepts will be evaluated using IGRIP. Visual aids produced from the CIT models will be used extensively to help communicate concepts to others. Information gained from the graphics models will assist XVRM designers in providing input on RM features to component designers at an early stage.

IGRIP allows "what ifs" to be evaluated inexpensively. It helps in assessing the problems associated with performing RM in a cluttered environment. IGRIP is not the ultimate RM tool. Hard mock-ups are still required to verify critical $R M$ hardware features. IGRIP cannot evaluate tolerances, weights, centers of gravity, and other important features. Graphics provides information to identify concepts that will not work, but it does not necessarily guarantee which concepts will work.

Graphic simulation of RM tasks is pyroving to be a significant compensation for 
the fusion equipment designer's lack of a remote handling experience base which, in the past, has been so necessary to properly visualize sequences of remote operations. The designer is now able to evaluate visually the functionality of a design option in a remote environment before committing to a final design.

\section{$\underline{\text { Reference }}$}

[1] D. Macdonald, "Maintenance concept development for the Compact Ignition Tokamak", in Proceedings of the ANS Third Topical Meeting on Remote Systems and Robotics, March 1989. 

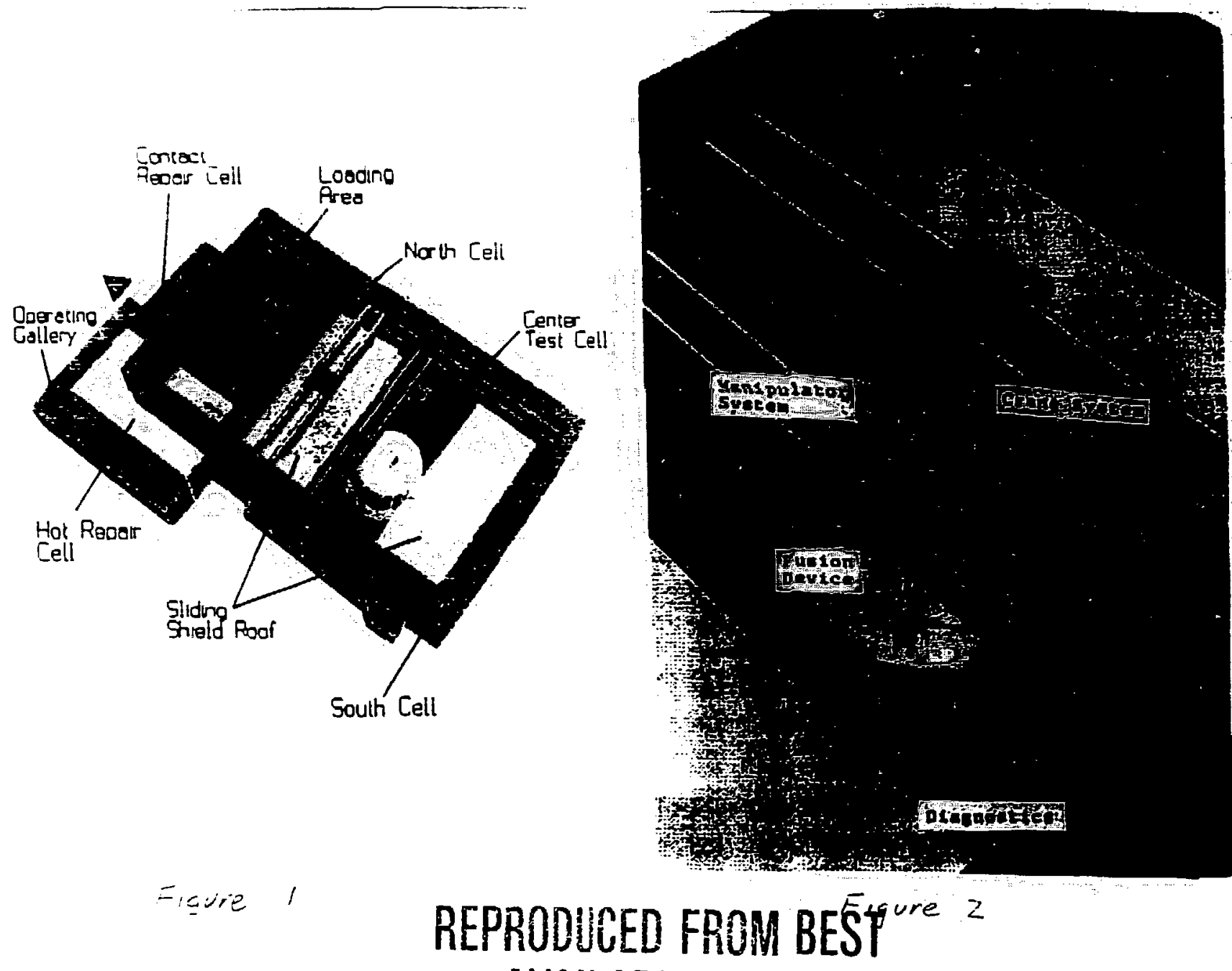

\section{AVAII ARIE COPY}

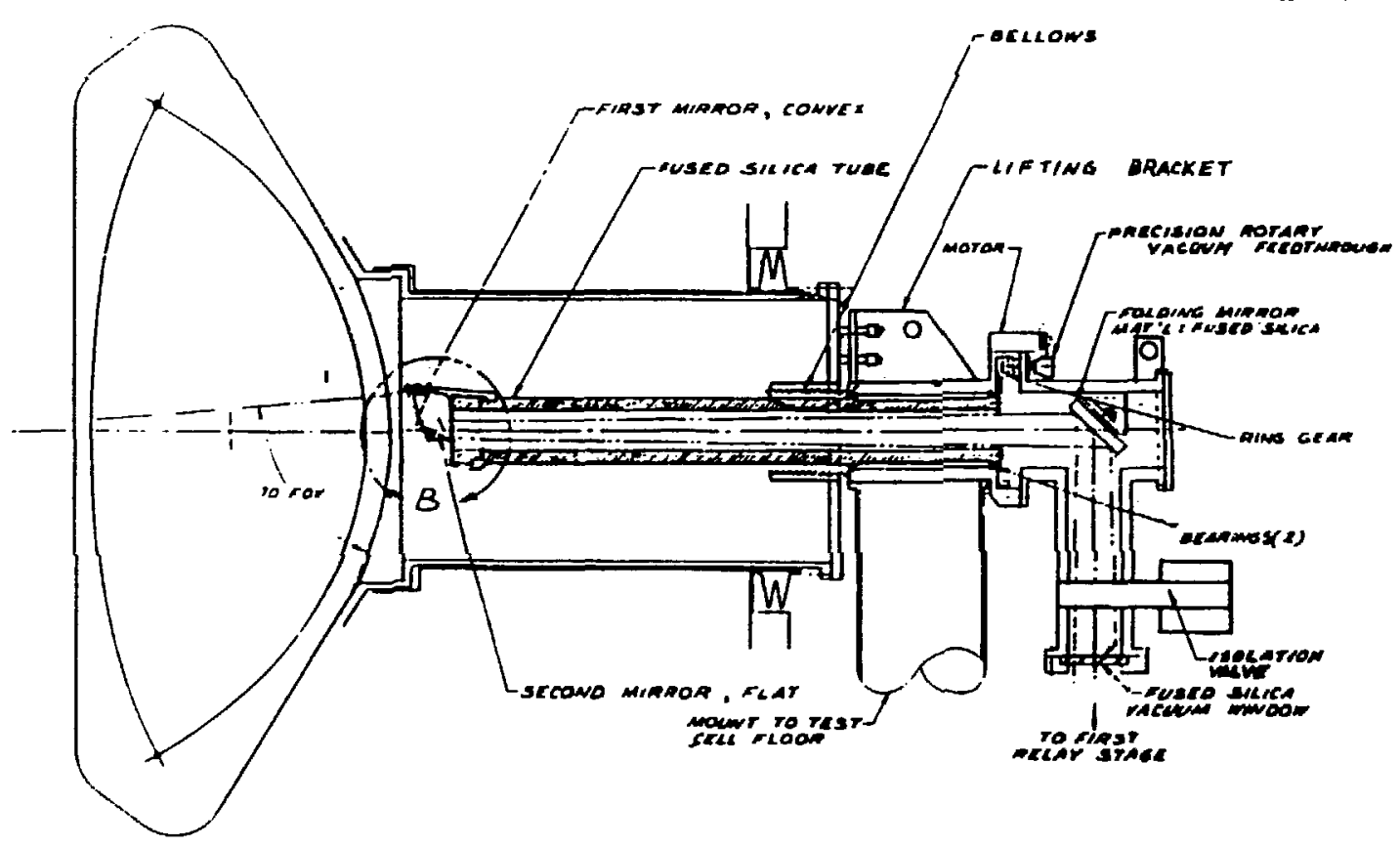

